\title{
OS PERIGOS SUBSUMIDOS NA CONTRACEPC̄̃O DE EMERGÊNCIA: MORALIDADES E SABERES EM JOGO*
}

Elaine Reis Brandão ${ }^{i}$

Universidade Federal do Rio de Janeiro - Brasil

Cristiane da Silva Cabral ${ }^{i i}$

Universidade de São Paulo - Brasil

Miriam Ventura ${ }^{i i i}$

Universidade Federal do Rio de Janeiro - Brasil

Sabrina Pereira Paiva ${ }^{i v}$

Universidade Federal de Juiz de Fora - Brasil

Luiza Lena Bastos ${ }^{v}$

Naira V. B. Vidal Oliveira ${ }^{v i}$

Iolanda Szabo vii

Universidade Federal do Rio de Janeiro - Brasil

Resumo: O tema da "contracepção de emergência” desperta bastante inquietação social no Brasil. O termo remete a noções como "risco de engravidar", "sexo desprotegido”, (ir)responsabilidade ou (ir)racionalidade prévia ao exercício sexual, contrariando normas sanitárias que postulam necessidade de proteção à gravidez $e$

\footnotetext{
* Nossos agradecimentos à Faperj (processo no 110.085/2012) e CNPq (processo n 481422/2012-9), que apoiaram a pesquisa.

i Contato: brandao@iesc.ufrj.br

ii Contato: cabralcs@usp.br

iii Contato: miriam.ventura@iesc.ufrj.br

iv Contato: sabrinappaiva@gmail.com

v Doutoranda em Saúde Coletiva. Contato: luizalena2@gmail.com

vi Contato: nairavbvoliveira@gmail.com

vii Contato: iolandaszabo@globo.com
} 
às doenças sexualmente transmissíveis nas práticas sexuais. Ao contrário da pílula anticoncepcional de uso regular, que conta com maior aceitação social, a contracepção de emergência desperta muitas controvérsias. Discute-se esse desconforto em relação ao método, a partir de pesquisa com farmacêuticos e balconistas de farmácia sobre o tema. Duas perspectivas analíticas são exploradas: a primeira é a estratégia que assinala perigos e potenciais riscos à saúde que esse contraceptivo provocaria, com vistas à regulação dos corpos femininos, em especial, jovens e pobres, onde a reprodução é temida. A segunda discute o agenciamento feminino que o uso da contracepção de emergência evidencia, perspectiva que pode estar ferindo hierarquias morais e sociais, de classe e de gênero.

Palavras-chave: contracepção de emergência, gênero, moralidades, sexualidade.

Abstract: Emergency contraception is a theme of great social unrest in Brazil. The term refers to notions such as "risk pregnancy", "unprotected sex", (ir)responsibility or (ir)rationality in face of sexual practice, which contradicts public health norms that claim the need for protection against pregnancy and sexually transmitted diseases. Unlikely the oral contraceptive pill of regular use, which has greater social acceptance among laypeople and experts, emergency contraception arises many controversies. We discuss this discomfort or demonization regarding the emergency contraception based on an anthropological research carried on with pharmacists and pharmacy sellers about this subject. This article discusses two mains analytical perspectives: the first is a kind of strategy that calls attention to the dangers and potential health risks that this contraceptive can have into women's bodies, especially into young and poor women's bodies, since there is great fear about reproduction in these groups. The second argues that the use of emergency contraception explicit female agency, perspective that diverges from some moral, cultural, social and gender hierarchies.

Keywords: emergency contraception, gender, moralities, sexuality.

\section{Introdução}

O tema da "contracepção de emergência" (CE) desperta bastante inquietação social no Brasil. Categoria adotada no meio científico/acadêmico para designar "pílula do dia seguinte", como é conhecida no senso comum, o próprio termo engendra a noção de "emergência", frequentemente associada no imaginário/universo da saúde pública às noções de "risco", "perigo", 
"epidemia", "acidente", entre outros que evocam certa preocupação com algo iminente que deve ser evitado ou combatido. No Dicionário Houaiss (2012), nos significados do termo "emergência" menciona-se "situação grave, perigosa, momento crítico ou fortuito", e sua etimologia destaca: "situação grave, contingência, combinação inesperada de circunstâncias imprevistas (ou o que delas resulta) e que exigem ação imediata". Decerto ambas as expressões - a nativa e a técnica - denotam a necessidade de uso do contraceptivo (levonorgestrel) após a relação sexual em tempo hábil para sua ação efetiva (até 120 horas). Mas é curioso que os sentidos subjacentes às duas expressões permaneçam latentes nas representações sociais existentes sobre o uso do método e suas consumidoras.

O termo remete ainda a noções como o "risco de se engravidar", o "sexo desprotegido", a (ir)responsabilidade ou (ir)racionalidade prévia ao exercício sexual, contrariando as normas sanitárias que postulam a necessidade de planejamento da gravidez e proteção às doenças sexualmente transmissíveis. Assim, ao contrário da pílula anticoncepcional de uso regular, diário, que conta na atualidade com ampla aceitação social entre leigos e experts ${ }^{1}$ (Dias, 2015; Pedro, 2010), chegando até mesmo a ser considerada uma "droga de estilo de vida" (Nucci, 2012; Watkins, 2012), a contracepção de emergência desperta muitas controvérsias (Foster; Winn, 2012; Prescott, 2011), embora seja também um método contraceptivo hormonal.

Neste artigo, pretendemos nos debruçar sobre esse desconforto ou mesmo certa demonização em relação à contracepção de emergência, considerando o contexto local da sociedade brasileira. Para tanto, tomaremos como inspiração de nossas reflexões material empírico derivado de uma pesquisa socioantropológica com farmacêuticos e balconistas de farmácia sobre o tema da contracepção de emergência.

\footnotetext{
Não se desconhece a vasta literatura que aborda a trajetória histórica e cultural da pílula hormonal e os muitos debates científicos e políticos que integram a história da contracepção e de seus dispositivos (Jütte, 2008; Marks, 2001; McLaren, 1996; Tone, 2001; Watkins, 1998). No entanto, para fins analíticos neste artigo, parte-se do pressuposto de que há hoje uma relativa aceitação social e incorporação desse dispositivo na área da saúde, para desenvolver o argumento em contraste da não aceitação social da contracepção de emergência, apesar de aprovada nas normas oficiais do Ministério da Saúde.
}

Horizontes Antropológicos, Porto Alegre, ano 23, n. 47, p. 131-161, jan./abr. 2017 


\section{Contexto da contracepcão de emergência no Brasil}

O acesso das mulheres à contracepção de emergência foi aprovado no Brasil em 1996 e o método foi incorporado às normas técnicas de assistência ao planejamento familiar do Ministério da Saúde (Brasil, 2002), inicialmente no regime Yuzpe, ou seja, pela combinação de pílulas anticoncepcionais orais comuns, à base de etinilestradiol e levonorgestrel. Ela também está disponível nos serviços públicos de prevenção e tratamento dos agravos resultantes da violência sexual contra mulheres e adolescentes desde 1998 (Brasil, 2011a). A Agência Nacional de Vigilância Sanitária (Anvisa) aprovou a comercialização do Postinor-2 (levonorgestrel) no país em 1999, mediante prescrição médica. Em 2005, o Ministério da Saúde publicou um guia de esclarecimento aos profissionais de saúde a respeito de sua utilização (Brasil, 2005) e, em 2006, lançou a Nota técnica sobre anticoncepção de emergência (Brasil, 2006), esclarecendo sobre o uso do levonorgestrel e suas indicações. Em 2012, o Protocolo de utilização do levonorgestrel na anticoncepção hormonal de emergência (Brasil, 2012) foi divulgado, ampliando as possibilidades de dispensação do medicamento na atenção básica, mediante prescrição do médico ou do enfermeiro, em unidades básicas de saúde, unidades de pronto-atendimento, unidades da Estratégia de Saúde da Família e centros de saúde.

A assistência à saúde sexual e reprodutiva de mulheres no Sistema Único de Saúde do Brasil está prevista na Política Nacional de Assistência Integral à Saúde da Mulher (Brasil, 2011b). As ações voltadas à contracepção estão organizadas no manual técnico Assistência em planejamento familiar (Brasil, 2002), que estabelece um conjunto de atividades educativas, de aconselhamento e clínicas para orientar as usuárias no tocante à escolha do método para prevenir a gravidez que melhor se adéque às suas necessidades e expectativas, conforme a fase do ciclo de vida em que se encontram e das suas condições de parceria sexual. Analisando os dados da última Pesquisa Nacional de Demografia e Saúde da Criança e da Mulher (PNDS), realizada em 2006, Perpétuo e Wong (2009) ressaltam uma ampliação do uso de anticoncepcionais no país em relação à pesquisa anterior de 1996, com redução significativa do recurso à laqueadura tubária, entre mulheres de 15 a 44 anos, em união conjugal. No entanto, a esterilização feminina $(25,9 \%)$ e a pílula oral $(27,4 \%)$ continuam sendo os dois métodos mais prevalentes na população.

Horizontes Antropológicos, Porto Alegre, ano 23, n. 47, p. 131-161, jan./abr. 2017 
Um percentual de $18,4 \%$ de mulheres desse segmento declarou não usar método algum para prevenir a gravidez.

No Brasil, 12\% das mulheres de 15 a 49 anos, sexualmente ativas, que já haviam utilizado um método contraceptivo moderno algum vez na vida, afirmaram ter usado a contracepção de emergência, segundo a PNDS de 2006 (Brasil, 2008). Esse percentual sobe para $23,2 \%$ se considerarmos apenas as mulheres não unidas. A PNDS 2006 aponta que o método passou a ocupar o quinto lugar entre os métodos contraceptivos modernos usados por todas as mulheres e é o terceiro dentre os métodos modernos usados por mulheres não unidas e ativas sexualmente (últimos 12 meses). O uso é maior entre as mais jovens, correspondendo a 10,4\% entre adolescentes de 15 a 19 anos que já utilizaram algum método moderno alguma vez na vida; $18,5 \%$ entre jovens de 20 a 24 anos e $16,9 \%$ entre jovens de 25 a 29 anos.

A oferta de métodos contraceptivos na rede pública de serviços de saúde não contempla a demanda existente, havendo um grande número de pessoas que se dirigem diretamente às farmácias comerciais privadas para comprar a pílula anticoncepcional de uso diário ou o contraceptivo de emergência, com preço médio aproximado de US\$ 6,00 (seis dólares americanos), sem necessariamente receber orientação prévia (Cabral, 2011; Paiva; Brandão, 2012). Segundo a PNDS 2006 (Brasil, 2008, p.141-2), as farmácias aparecem como fonte de obtenção de métodos contraceptivos modernos para 42,5\% das usuárias de 15 a 49 anos, reunindo os métodos hormonais (pílula e injeção) e preservativo masculino. Tal via de acesso representa uma alternativa importante, já que os estabelecimentos farmacêuticos permanecem abertos à noite e fins de semana, facilitando a obtenção do contraceptivo em tempo oportuno, além de possuírem profissionais farmacêuticos para o atendimento aos consumidores. Embora haja recomendação da Anvisa de que a venda do contraceptivo de emergência seja feita mediante prescrição médica, tal norma não é respeitada no país. Há uma série de medicamentos vendidos nas farmácias sem atenderem devidamente a regulação sanitária vigente. A prática da automedicação na população brasileira é significativa (Arrais et al., 1997; Domingues et al., 2015; Naves et al., 2010).

$\mathrm{Na}$ literatura científica sobre o tema da contracepção de emergência no Brasil, não há estudos sobre sua dispensação farmacêutica. A partir do levantamento da literatura nacional e internacional, verificou-se que a produção científica brasileira carecia de estudos que abordassem os espaços das farmácias e drogarias, tendo em vista a vasta produção existente sobre os serviços 
públicos de saúde. A revisão de literatura científica internacional sobre as políticas de provisão da contracepção de emergência, notadamente por meio das farmácias, apontou duas perspectivas analíticas: a disponibilidade e as barreiras à contracepção de emergência nas farmácias, situando a posição dos farmacêuticos sobre o método; e a posição das usuárias/consumidoras do método a respeito do contraceptivo e seus locais de provisão (Paiva; Brandão, 2012). Tais estudos evidenciaram que a adoção de políticas públicas favoráveis à distribuição/venda da contracepção de emergência pelas farmácias recebe, de modo geral, avaliação positiva de farmacêuticos e usuários, principalmente devido à possibilidade de dispensar o contraceptivo de forma mais ágil. Os países da América do Norte e Europa que adotaram tal política discutem a necessidade de aconselhamento em saúde sexual e reprodutiva para os consumidores no âmbito da própria farmácia. No Brasil, até o momento não há política pública que inclua a provisão do método pelas farmácias/drogarias, como ocorre no contexto internacional.

A lei $\mathrm{n}^{0} 5.991$, de 17 de dezembro de 1973, estabelece que o processo de dispensação de medicamentos nas farmácias está sob a responsabilidade do profissional farmacêutico (Brasil, 1973). No entanto, na prática cotidiana do comércio farmacêutico no país, o balconista é, em geral, o funcionário responsável por vender os medicamentos, mantendo contato mais estreito com os consumidores do que os farmacêuticos. O balconista de farmácia é, em sua maioria, um profissional com escolaridade até o ensino médio, sem conhecimentos específicos sobre uso de medicamentos, a não ser pelo contato com material de divulgação dos laboratórios farmacêuticos. A penetração dos processos de medicalização (Conrad, 2007) e de "farmacologização" da sociedade (Williams; Martin; Gabe, 2011) amplia cada vez mais as relações diretas entre a indústria farmacêutica e seus consumidores, profissionais de saúde e vendedores.

Estudos realizados na área da sexualidade e do gênero apontam o quão difícil tem sido a gestão da sexualidade e da contracepção no par heterossexual, as dificuldades de uso regular de métodos anticoncepcionais, com ocorrência de gravidez imprevista e aborto em condições inseguras ${ }^{2}$ para a

\footnotetext{
2 De acordo com o decreto-lei no 2848/40 (Código Penal), o aborto é, via de regra, uma prática ilegal no Brasil. Não se pune o aborto praticado por médico se não há outro meio de salvar a vida da gestante ou se a gravidez resulta de estupro e o aborto é precedido de consentimento da gestante ou, quando incapaz, de seu representante legal (Brasil, 1940).
}

Horizontes Antropológicos, Porto Alegre, ano 23, n. 47, p. 131-161, jan./abr. 2017 
saúde das mulheres (Bajos et al., 2002, 2003; Cabral, 2012; Gonçalves et al., 2011; Luker, 1975; Spencer et al., 2014; Teixeira el al., 2012). As dificuldades de negociação entre os casais sobre o uso do preservativo masculino e/ou de outro método de proteção à gravidez, devido à forte hierarquia de gênero e uma cultura sexual que preconiza a "naturalidade" e a espontaneidade do ato sexual, impedem o planejamento prévio dos encontros sexuais, admitindo-se um certo grau de incerteza e do risco de se engravidar (Bozon; Heilborn, 2006; Cabral, 2011). Em tal contexto sociocultural, torna-se muito importante a disponibilidade da contracepção de emergência como um recurso oportuno àqueles que porventura tenham tido uma relação sexual sem proteção, seja de modo consensual com o parceiro ou sob constrangimento e uso de violência.

Investigações anteriores sobre o tema da contracepção de emergência no contexto latino-americano e brasileiro (Bergallo, 2010; Costa et al., 2008; Díaz et al., 2003a, 2003b; Faúndes et al., 2007; Hardy et al., 2001; Martin, A., 2004; Pecheny; Tamburrino, 2009), assim como no cenário internacional (Shoveller et al., 2007; Simonds; Ellertson, 2004), apontam barreiras e dificuldades de implementação das políticas públicas de distribuição da contracepção de emergência. Há preconceito, discriminação, desinformação e valores morais e religiosos dos profissionais de saúde e gestores públicos que recriminam o uso do contraceptivo de emergência por considerá-lo abortivo ou por não aprovarem o livre exercício sexual das mulheres como um direito, subjugando-o ao casamento.

\section{A pesquisa empreendida}

A pesquisa designada "Uma investigação socioantropológica em farmácias: posição de farmacêuticos e balconistas sobre a contracepção de emergência", desenvolvida entre os anos de 2012-2014, buscou conhecer a visão de profissionais que trabalham em farmácias privadas no Brasil sobre o tema da contracepção de emergência. $O$ marco teórico que orienta a investigação postula que a compreensão sociológica dos fenômenos relativos à sexualidade e à reprodução precisa ser contextualizada no âmbito das relações sociais estabelecidas pelos sujeitos em suas interações cotidianas (Bozon, 2002; Gagnon, 2006; Weeks, 2000). Nessa perspectiva teórico-metodológica, busca-se conhecer as representações e práticas sociais dos sujeitos em relação às 
suas escolhas contraceptivas, dos profissionais de saúde e dos educadores que desenvolvem ações para fomentar e apoiar tais cuidados, no âmbito das políticas públicas, tendo como esteio a matriz da sociologia compreensiva (Berger, Luckmann, 1978; Watier, 2002). Assim, opiniões, atitudes, comportamentos de qualquer sujeito só podem ser compreendidos se devidamente articulados a sua visão de mundo mais ampla, a seu universo de valores e crenças morais, segundo sua posição de classe, gênero, raça/etnia, filiação religiosa, geração, e socialização em determinado ambiente específico, dentre outras marcas sociais que condicionam seu olhar para o mundo.

A questão de pesquisa que ensejou tal estudo foi a necessidade de conhecer as circunstâncias sociais que condicionam o acesso das mulheres à contracepção de emergência nas farmácias privadas, mediado pela interação com farmacêuticos e balconistas. Já se conhece as muitas dificuldades que cercam o acesso das mulheres ao método na rede pública de serviços de saúde, em razão de preconceitos e juízos de valor dos profissionais de saúde sobre a sexualidade feminina. Tendo em vista a busca maciça da contracepção de emergência no comércio farmacêutico, intencionava-se saber se os mesmos constrangimentos descritos em relação aos serviços públicos de saúde também existiam naquele espaço, e como se dava tal interação, descrevendo-se como as representações sociais desses profissionais estariam intervindo em suas práticas profissionais.

No país, há duas categorias principais de funcionários que trabalham em farmácias: o farmacêutico, profissional graduado nas universidades públicas ou privadas, e os vendedores de medicamentos propriamente ditos, conhecidos como "balconistas", em geral, menos escolarizados que os farmacêuticos. A pesquisa atual foi precedida por um estudo etnográfico sobre a comercialização da contracepção de emergência, realizado em farmácia da zona norte da cidade do Rio de Janeiro, com início em 2010 (Paiva, 2014). Os primeiros resultados dessa etnografia foram determinantes para o desenho do estudo subsequente. Após examinar detidamente o universo social dos profissionais que atuam no espaço de uma drogaria privada de médio porte, a saber, balconistas, farmacêuticos, gerentes, representantes de laboratórios farmacêuticos e proprietário de rede de drogarias (Paiva, 2014), optamos por ampliar o alcance da investigação seguinte e incorporar profissionais de diferentes contextos socioculturais, ampliando a consulta a outros estados da federação. 
Contou-se assim com estratégias diferenciadas para a coleta de dados entre as duas categorias de trabalhadores de farmácia: um questionário on-line, estruturado, autoaplicado, anônimo, disponível na Plataforma DataSUS/ FormSUS para os farmacêuticos, e entrevistas face a face que utilizaram um roteiro semiestruturado de questões com um conjunto de 20 balconistas que atuam em estabelecimentos da região metropolitana do Rio de Janeiro: 12 homens e 8 mulheres.

A opção em utilizar um instrumento fechado para coleta de dados junto aos farmacêuticos em uma pesquisa socioantropológica mais ampla visava a possibilidade de aceder a um número maior de informantes dessa categoria profissional, de modo a conhecer de forma mais abrangente o conjunto de representações e práticas sociais desses profissionais no tocante à dispensação da contracepção de emergência.

O convite aos farmacêuticos para participação na pesquisa foi divulgado em parceria com órgãos de classe da categoria profissional, através de websites, revistas e envio de mala direta pelos Conselhos Regionais de Farmácia dos estados do Rio de Janeiro e de São Paulo, Conselho Federal de Farmácia e a Associação Brasileira de Farmácia. A mensagem de chamada era claramente dirigida aos profissionais que atuavam especificamente no ramo de farmácias e drogarias.

O questionário continha perguntas fechadas, distribuídas em cinco módulos: 1) dados sociodemográficos dos respondentes; 2) dinâmica de trabalho na farmácia onde atuam; 3) opiniões sobre a "pílula do dia seguinte"; 4) atendimento ao consumidor da "pílula do dia seguinte"; 5) avaliação sobre o questionário. Em outubro de 2012, foi realizada uma etapa-piloto, para teste do instrumento, junto a 114 farmacêuticos residentes no estado do Rio de Janeiro, participantes de um treinamento promovido pela rede de drogarias onde atuam. Nessa ocasião, o questionário foi respondido manualmente por eles, sendo $75,7 \%$ desse público compostos de profissionais mulheres. A maioria $(94,6 \%)$ era de responsáveis técnicos nos estabelecimentos em que trabalhavam. Aproximadamente $70 \%$ deles haviam se formado nos últimos seis anos. Após as reformulações necessárias e obtenção da versão final do instrumento, procedeu-se a coleta de dados on-line, entre março e maio de 2013. O formulário podia ser acessado através de um link mediante adesão voluntária dos interessados, que assinalaram concordância ao Termo de Consentimento Livre e Esclarecido. Assim, o conjunto dos respondentes compõe uma amostra não 
probabilística $(\mathrm{n}=383)$, configurando o que usualmente se denomina de amostra acidental ou de contingência (Pires, 2012). Nesse trabalho, a análise dos dados incluiu a distribuição de frequências relativas e o cruzamento de variáveis para a análise de aspectos de interesse. Utilizamos o teste de qui-quadrado para comparar as proporções obtidas em relação às opiniões dos farmacêuticos segundo as estratificações examinadas (sexo, faixa etária, origem/estado da federação em que atuam). Utilizou-se o pacote estatístico do SPSS (Statistical Package for the Social Sciences - versão 17) para compilação e análise dos dados.

As entrevistas com os balconistas de farmácia ocorreram entre os meses de agosto de 2012 e maio de 2013. Foram realizadas por três pesquisadoras com formação em ciências sociais, com treinamento nos temas da sexualidade, do gênero, da saúde reprodutiva e da contracepção para conseguirem abordá-los com naturalidade junto aos sujeitos. O roteiro das questões da entrevista foi organizado em quatro módulos: 1) perfil sociodemográfico dos entrevistados; 2) venda da contracepção de emergência e interação social com as consumidoras no balcão da farmácia; 3) opinião sobre a contracepção de emergência e sua regulação sanitária no Brasil; 4) experiência de uso pessoal da contracepção de emergência.

Para a seleção dos balconistas a serem entrevistados, adotou-se inicialmente a estratégia de rede de indicações (Pires, 2012), em que o primeiro balconista entrevistado indica outro para ser contatado pela equipe de pesquisa, preferencialmente de farmácia e região distintas da sua, para se captar a diversidade de experiências sociais e profissionais, e assim sucessivamente. Entretanto, houve dificuldades para se obter a totalidade dos 20 entrevistados por essa estratégia de captação, devido à indisponibilidade de tempo para a entrevista entre os sujeitos contatados. Em geral, eles têm uma excessiva jornada de trabalho nas farmácias, superior a 40 horas semanais, além de muitos se dedicarem aos estudos e cursos nos horários noturnos. Assim, no trabalho de campo, também foi adotada uma abordagem mais direta, com visitas das pesquisadoras a diferentes estabelecimentos farmacêuticos para contato direto e convite à participação na pesquisa.

Utilizou-se o Termo de Consentimento Livre e Esclarecido, documento que esclarece o entrevistado sobre os objetivos da pesquisa e atesta seu consentimento em participar do estudo. As entrevistas foram registradas em gravador digital, tendo sido realizadas principalmente no espaço da própria 
farmácia (13), devido à indisponibilidade dos balconistas de se ausentarem do trabalho durante o expediente e de conseguirem horários extras para as entrevistas, ou em suas proximidades (7), como em parques ao ar livre, cafés e lanchonetes, antes da entrada ou após a saída dos entrevistados dos seus respectivos locais de trabalho. Apesar de algumas entrevistas terem sido realizadas durante o expediente de trabalho dos informantes, recurso que poderia se constituir em uma limitação para a livre expressão de seu discurso, devido à menor privacidade, a maioria dos entrevistados não demonstrou receio ou intimidação. Ao contrário, insistiram na possibilidade da entrevistadora poder conversar sobre o tema da venda da contracepção de emergência naquele espaço. Inicialmente a equipe de pesquisa resistiu a essa proposta vinda dos balconistas contatados, mas com o tempo se compreendeu que eles "naturalizavam" a entrevista de pesquisa como mais uma "conversa no balcão", dentre tantas que ocorriam em seu cotidiano de atendimento ao público na farmácia. Assim, nessas circunstâncias, as entrevistas tiveram duração mais breve, média de 30 minutos, e o contato entre entrevistadora/entrevistado/a foi menos estreito.

Para a análise dos dados empíricos coletados, houve a transcrição literal das entrevistas na íntegra, pelas próprias pesquisadoras responsáveis, para um arquivo digital, sem identificação nominal dos participantes, para preservar o sigilo, a confidencialidade e o anonimato dos sujeitos. Um diário de campo das circunstâncias sociais que cercaram a entrevista, desde o contato inicial com o entrevistado, por intermédio de indicação de amigos ou por visita à farmácia, até a realização do encontro propriamente dito, as reações e atitudes dos participantes durante a conversa com as pesquisadoras, todas as observações foram devidamente registradas para se somarem aos discursos nativos dos balconistas. Houve a leitura intensiva e repetitiva do material empírico (entrevistas transcritas e diários de campo) por duas pesquisadoras mais experientes da equipe de pesquisa, uma delas tendo realizado estudo etnográfico em farmácia do Rio de Janeiro para sua tese de doutorado sobre o tema (Paiva, 2014). Buscou-se captar os elementos recorrentes e relevantes nos discursos dos entrevistados, para se extrair as categorias temáticas mais expressivas e significativas da visão de mundo dos participantes (Kornblit, 2007; Minayo, 2012; Schutz, 1979; Silverman, 2000).

As narrativas dos sujeitos entrevistados trazem mais semelhanças do que divergências entre eles. Ao compararmos o material empírico extraído 
da pesquisa com o produzido por Paiva (2014), em investigação etnográfica realizada em farmácia da zona norte da cidade do Rio de Janeiro, foi possível constatar vários pontos em comum no que se refere a um certo ethos do balconista, sua maneira peculiar de pensar e atuar em relação ao público atendido.

O projeto de pesquisa foi aprovado na Plataforma Brasil/Comitê de Ética em Pesquisa do Instituto de Estudos em Saúde Coletiva da Universidade Federal do Rio de Janeiro (IESC/UFRJ), em 3 de outubro de 2012, com parecer $n^{\circ} 114.610$ (CAAE: 04918212.9.0000.5286), tendo cumprido todas as normas éticas da resolução vigente na ocasião.

\section{Representacõos da contracepscão de emergência: sobre excessos e descontroles}

Para subsidiar a discussão aqui proposta, optamos por apresentar alguns resultados da consulta aos farmacêuticos, extraídos em especial do módulo "Atendimento ao consumidor da pílula do dia seguinte", que aborda as representações dos entrevistados sobre as mulheres consumidoras da contracepção de emergência e aspectos da interação social entre ambos no balcão da farmácia.

Do total de 383 farmacêuticos que voluntariamente atenderam à convocação da pesquisa, $74,5 \%$ são do sexo feminino e $25,5 \%$, do masculino. A maioria deles, homens e mulheres, tem até 30 anos de idade $(61,3 \%)$, constituindo um grupo jovem de profissionais atuando no âmbito das farmácias. A idade mediana entre os homens é de 30 anos e entre as mulheres, 28 anos. Eles são majoritariamente brancos (71,7\%); 20,4\% dos homens e das mulheres se declaram pardos e aproximadamente $5 \%$, pretos, totalizando $25,2 \%$ daqueles considerados negros. Mais da metade dos farmacêuticos se declaram católicos. O percentual de homens que declara não ter ou não frequentar religião é de $22,7 \%$; dentre as mulheres o valor é aproximadamente a metade do encontrado para eles $(10,4 \%)$. Quanto a frequentar outras religiões, essa relação se inverte, com as mulheres obtendo um percentual de $22,5 \%$ e os homens, de $11,3 \%$.

Aproximadamente $65 \%$ dos participantes estão formados há no máximo cinco anos; os que têm entre cinco e dez anos de formados representam aproximadamente $20,0 \%$. Cerca de $80 \%$ dos informantes atuam como responsáveis técnicos (RT) no estabelecimento em que se encontram no momento da 
pesquisa. Quanto à sua distribuição no país, a região Sudeste do Brasil congrega $64,9 \%$ dos respondentes, oriundos majoritariamente dos estados do Rio de Janeiro (30,3\%) e de São Paulo (27,4\%), e em terceiro lugar figuram os que atuam na região $\mathrm{Sul}(16,4 \%)$. Os farmacêuticos residentes nas outras três regiões do país (Norte, Nordeste e Centro-Oeste) somam 18,7\%. Pode-se afirmar que uma parcela significativa dos informantes é composta por mulheres brancas, católicas, com até 30 anos, e possuindo até cinco anos de formadas em faculdades de farmácia privadas, situadas na região Sudeste. O fato de haver $2 / 3$ de mulheres respondentes pode estar associado não apenas ao fenômeno da crescente feminização da profissão, aspecto já destacado por algumas investigações (Carrillo-García et al., 2013; Machado et al., 2006), mas também a uma maior sensibilização e interesse dentre as mulheres pelo próprio tema do estudo. De modo geral, a contracepção é fortemente representada como um "assunto de mulheres".

Indagados sobre o perfil do consumidor que recorre à farmácia para comprar a contracepção de emergência, a representação dominante é a de que se trata de um grupo de mulheres $(72,9 \%)$, jovens $(97,6 \%)$, com idades entre 16 e 30 anos, que utilizam a contracepção de emergência de modo regular $(65,6 \%)$, em situações emergenciais $(31,5 \%)$, que não compram o medicamento de forma antecipada $(9,6 \%)$ mas após a relação sexual desprotegida $(90,4 \%)$. Segundo informam os farmacêuticos, elas se dirigem ao balcão da farmácia para solicitar a contracepção de emergência sentindo-se constrangidas $(50,8 \%)$ ou desconfiadas $(13,8 \%)$, enquanto $20,9 \%$ sentem-se à vontade e $14,6 \%$ apresentam-se indiferentes com o contexto da compra. Para os entrevistados, os principais motivos para o uso da contracepção de emergência podem ser agrupados em dois blocos: aqueles que denotam um comportamento feminino não razoável/racional ou previdente e os que revelam constrangimentos de gênero na relação afetivo-sexual. Dentre os primeiros temos: as mulheres que não utilizam outra forma de proteção $(78,4 \%)$, esquecem de usar o método hormonal de rotina $(59,7 \%)$, por displicência $(56,1 \%)$ ou por imediatismo no ato sexual (45\%), por preguiça de usarem outro método $(34,7 \%)$ e insegurança quanto ao método adotado (33,4\%). Em relação aos constrangimentos de gênero, aparece rompimento do preservativo masculino (70,5\%) e recusa masculina do uso do preservativo (47,6\%). Um outro motivo apontado também merece atenção: o fato de as mulheres não terem relações sexuais frequentes $(25,3 \%)$. 
Tabela 1. Representações dos farmacêuticos em relação ao perfil dos/as consumidores/as da contracepção de emergência segundo sexo do informante.

\begin{tabular}{|c|c|c|c|}
\hline \multirow{2}{*}{ Representações sobre perfil dola consumidor/a } & \multicolumn{2}{|c|}{ SEXO } & \multirow{2}{*}{ TOTAL } \\
\hline & Masculino & Feminino & \\
\hline \multicolumn{4}{|l|}{ A pílula do dia seguinte está sendo utilizada: } \\
\hline somente em casos emergenciais & $26,8 \%$ & $33,1 \%$ & $31,5 \%$ \\
\hline como contraceptivo regular & $68,0 \%$ & $64,8 \%$ & $65,6 \%$ \\
\hline não sabe & $5,2 \%$ & $2,1 \%$ & $2,9 \%$ \\
\hline \multicolumn{4}{|l|}{ As pessoas compram a pílula do dia seguinte, em geral: } \\
\hline de forma antecipada & $9,3 \%$ & $9,7 \%$ & $9,6 \%$ \\
\hline somente após relação sexual desprotegida & $90,7 \%$ & $90,3 \%$ & $90,4 \%$ \\
\hline \multicolumn{4}{|l|}{ Quem mais compra a pílula do dia seguinte? } \\
\hline mulher & $69,1 \%$ & $74,2 \%$ & $72,9 \%$ \\
\hline \multicolumn{4}{|c|}{$\begin{array}{l}\text { A faixa etária do público consumidor deste medicamento é } \\
\text { preferencialmente: }\end{array}$} \\
\hline menor de 15 anos & $2,1 \%$ & $1,4 \%$ & $1,6 \%$ \\
\hline entre 16 e 20 anos & $47,4 \%$ & $49,8 \%$ & $49,2 \%$ \\
\hline entre 21 e 30 anos & $50,5 \%$ & $47,7 \%$ & $48,4 \%$ \\
\hline 31 anos ou mais & - & $1,1 \%$ & $0,8 \%$ \\
\hline \multicolumn{4}{|c|}{ Ao solicitarem no balcão a PDS, os consumidores sentem-se: } \\
\hline à vontade & $24,7 \%$ & $19,6 \%$ & $20,9 \%$ \\
\hline envergonhados & $48,5 \%$ & $51,6 \%$ & $50,8 \%$ \\
\hline desconfiados & $17,5 \%$ & $12,5 \%$ & $13,8 \%$ \\
\hline indiferentes & $9,3 \%$ & $16,4 \%$ & $14,6 \%$ \\
\hline \multicolumn{4}{|l|}{ Principais motivos para o uso da PDS:* } \\
\hline falha do anticoncepcional de rotina & $23,7 \%$ & $27,6 \%$ & $26,6 \%$ \\
\hline esquecimento de usar método hormonal & $61,9 \%$ & $59,0 \%$ & $59,7 \%$ \\
\hline insegurança quanto ao MAC adotado & $36,1 \%$ & $32,5 \%$ & $33,4 \%$ \\
\hline não utilizar outra forma de proteção & $87,6 \%$ & $75,3 \%$ & $78,4 \%$ \\
\hline camisinha estourar & $66,0 \%$ & $72,1 \%$ & $70,5 \%$ \\
\hline recusa do uso do preservativo & $50,5 \%$ & $46,6 \%$ & $47,6 \%$ \\
\hline não ter relações sexuais frequentes & $18,6 \%$ & $27,6 \%$ & $25,3 \%$ \\
\hline não poder utilizar outro método & $7,2 \%$ & $7,8 \%$ & $7,6 \%$ \\
\hline displicência & $53,6 \%$ & $56,9 \%$ & $56,1 \%$ \\
\hline preguiça de usar outro método & $33,0 \%$ & $35,3 \%$ & $34,7 \%$ \\
\hline imediatismo no ato sexual & $56,7 \%$ & $41,0 \%$ & $45,0 \%$ \\
\hline promiscuidade & $34,0 \%$ & $23,0 \%$ & $25,8 \%$ \\
\hline falta de informação sobre MAC & $29,9 \%$ & $24,0 \%$ & $25,5 \%$ \\
\hline
\end{tabular}

Fonte: pesquisa "Uma investigação socioantropológica em farmácias: posição de farmacêuticos e balconistas sobre a contracepção de emergência".

* Neste item havia a possibilidade de assinalar mais de uma resposta, por isso esta parte da tabela não totaliza $100 \%$. 
A maioria dos entrevistados $(78,1 \%)$ afirmam que os consumidores apresentam dúvidas sobre a contracepção de emergência e seu uso e que costumam ser procurados, na curta interação existente no balcão da farmácia, para tirar dúvidas a respeito. Também costumam oferecer informações aos consumidores, mesmo sem serem interpelados $(61,6 \%)$, sentindo-se seguros para fazê-lo $(73,4 \%)$. Eles consideram importante informar sobre a posologia do medicamento $(66,3 \%)$ no momento de sua venda. Assim como os balconistas entrevistados, os farmacêuticos são contrários à venda $\mathrm{OTC}^{3} \mathrm{da}$ contracepção de emergência (72,4\%). A estratégia de venda OTC configura a possibilidade de acesso a qualquer produto (inclusive medicamento) de modo mais livre, pois os mesmos ficam disponíveis nas gôndolas das farmácias, podendo ser adquiridos pelo consumidor sem a necessária mediação do funcionário.

Por fim, no que tange aos resultados do módulo "Opiniões sobre a pílula do dia seguinte", alguns aspectos para reflexão devem ser destacados. A maioria dos farmacêuticos entrevistados $(89,8 \%)$ respondeu que a contracepção de emergência é um medicamento muito utilizado; $87,7 \%$ afirmam que ele é um medicamento perigoso para a saúde da mulher, se utilizado de forma regular. A contracepção de emergência não é considerada abortiva pela maioria (71,5\%) dos respondentes, em contraste com a opinião positiva de $21,9 \%$ dos farmacêuticos e de $6,5 \%$ que não souberam responder. No entanto, em sua grande maioria $(89,9 \%)$, nossos informantes consideram que a contracepção de emergência é uma "bomba hormonal". Fizemos uso dessa categoria no estudo tendo em vista que esta é uma acepção comumente usada no contexto brasileiro em relação à contracepção de emergência, disseminada não só entre pessoas leigas, mas também entre profissionais de saúde, sites de internet, veículos de comunicação de mídia, etc. A ideia de "bomba hormonal" parece enfatizar o "excesso" de hormônios e o potencial "perigo" explosivo representado pela contracepção de emergência aos corpos femininos. Voltaremos adiante a esse aspecto.

\footnotetext{
Behind the counter (BTC), atrás do balcão, com aquisição do produto pela mediação de funcionários; ou venda over the counter (OTC), com acesso livre do consumidor direto ao produto, disponibilizado nas gondolas da drogaria.
} 


\section{0 "mal-estar" da contracepsão}

À luz do material empírico produzido na pesquisa e de uma expressiva literatura que discute o tema no país, vislumbram-se duas possibilidades de interpretação desse mal-estar que cerca a trajetória da difusão da contracepção de emergência nesses últimos 20 anos. Tais vias de reflexão não são concorrentes entre si, ao contrário, elas podem se combinar de modo complementar.

\section{Os "perigos" da contracepsão de emergência}

A primeira consiste na identificação de uma estratégia disciplinar subliminar à ênfase recorrente aos perigos da contracepção de emergência. Ao se realçar, assinalar, ampliar os perigos e potenciais riscos à saúde que esse contraceptivo provocaria, coloca-se em discussão a regulação dos corpos. Estamos falando de corpos femininos, mas principalmente de corpos de mulheres jovens e pobres, lócus onde a reprodução é mais temida. Sob um manto aparente de preocupações sanitárias com a saúde feminina, o que se teme de fato é o pleno exercício sexual entre mulheres, jovens e pobres, que redundaria em "maternidades de risco", tão bem discutidas por Faya Robles (2015). Tal estratégia pode ser percebida em concepções presentes na mídia (Brandão, 2016; Souza; Brandão, 2012), em discursos de especialistas e profissionais de saúde e de farmácia que, amparados por mecanismos disciplinares de regulação dos corpos e da sexualidade (Clarke, 1998; Foucault, 1999; Martin, E., 2006), tão caros às instituições de saúde, explicitam em suas práticas profissionais - seja nos serviços públicos de saúde ou nos estabelecimentos farmacêuticos - os temores quanto aos perigos ou prejuízos que o medicamento pode trazer às mulheres.

Resumidamente, tais perigos condensam-se em duas estratégias discursivas que podem adquirir o status de uma acusação a quem o utiliza: que tenha efeitos abortivos e/ou que seja uma "bomba hormonal". Há aqui um deslocamento importante que precisa ser abordado. Destaca-se que sendo o aborto proibido no Brasil ${ }^{4}$ e em diversos países da América Latina, há forte oposição

4 Ver nota 2.

Horizontes Antropológicos, Porto Alegre, ano 23, n. 47, p. 131-161, jan./abr. 2017 
de segmentos religiosos de diferentes confissões (católicos, protestantes, espíritas, dentre outros) ao direito das mulheres de interromperem sua gravidez. Por ser um contraceptivo utilizado após o ato sexual, há complexos debates para definir como seu mecanismo de ação pode ou não afetar o feto, em caso de efetiva fecundação. Tais credos absorvem a resistência ao contraceptivo de emergência na luta mais ampla antiaborto, transformando-o equivocadamente em um medicamento abortivo, em relação a outros como o misoprostol. Nesse cenário de disputas políticas, científicas e religiosas, diversos organismos internacionais e nacionais de saúde vêm a público atestar a segurança, eficácia e oportunidade da contracepção de emergência, assegurando sua indicação para prevenir a gravidez e sua inocuidade em caso de ter havido a fecundação (Brasil, 2005; World Health Organization, 2010, 2012). Observa-se nos últimos anos um certo enfraquecimento da hipótese abortiva da contracepção de emergência; no entanto, tal apelo ainda se faz presente tanto entre a população em geral quanto entre profissionais de saúde, aspecto refletido nas respostas de farmacêuticos e balconistas em nossa pesquisa. Possivelmente a ampliação do debate público sobre o tema, as estratégias de advocacy em torno da melhoria do acesso ao contraceptivo de emergência, promovidas pelos Consórcios Internacional (ICEC) e Latino-Americano de Anticoncepção de Emergência (CLAE), voltadas para "países em desenvolvimento" (Bastos, 2015), estejam mobilizando atores políticos tão centrais quanto diversos, a exemplo de agências reguladoras sanitárias, grupos feministas, laboratórios farmacêuticos, que congregam esforços na direção de facilitar a aceitação social do contraceptivo, isentando-o de seus temidos efeitos abortivos.

Concomitante a um certo refluxo da hipótese abortiva, parece aumentar uma certa preocupação ou temor de que a contracepção de emergência seja concebida como uma "bomba hormonal", argumento inicialmente combinado ao seu potencial efeito abortivo, mas atualmente deslocado e melhor articulado à tese da preeminência de um "corpo hormonal" (Harding, 1996; Oudshoorn, 1994; Rohden, 2008). A categoria "bomba hormonal", também utilizada com ênfase social condenatória, desloca os possíveis efeitos deletérios da contracepção de emergência para o âmbito das desordens hormonais, da alteração de um ciclo hormonal "natural", o que afetaria a saúde das mulheres. Uma dose de hormônios considerada excessiva e concentrada para administração única ("alta dosagem", "bomba") seria destrutiva aos corpos femininos. Novamente, o argumento da desordem hormonal se desloca para 
o medo ou pânico de uma suposta desordem moral, combinando estratégias de regulação e disciplinamento dos corpos e sexo femininos. A expressão "bomba hormonal" parece combinar elementos que precisam ser mais bem elucidados. Por um lado, ela reatualiza concepções advindas da cultura popular que relaciona a ingestão de pílulas anticoncepcionais em grande quantidade - uma "overdose hormonal" (Leal, 1994), com intuito de "baixar as regras" ou fazer descer a menstruação. De outro, segmentos contrários ao aborto que a difundem propositadamente como um medicamento abortivo enfatizam seu caráter de "bomba" para assustar e distanciar potenciais usuárias, temerosas dos riscos inerentes às práticas abortivas inseguras. Por fim, a noção de "bomba hormonal" carrega influências da literatura sobre o "corpo hormonal" (Harding, 1996; Oudshoorn, 1994; Rohden, 2008), deslocando do campo religioso para o terreno da ciência, em especial da endocrinologia, os eventuais perigos da contracepção de emergência. Tal deslocamento representa uma significativa mudança nos argumentos que sustentam a reprovação da contracepção de emergência. Não se trata de crença religiosa, mas de fé na ciência. Não é casual que os farmacêuticos, assim como balconistas, tenham respondido negativamente ao fato de ela ser abortiva e afirmativamente ao fato de ela ser uma "bomba hormonal". Há aqui crenças dos efeitos deletérios aos corpos femininos, expressos por exemplo nos seguintes depoimentos de alguns balconistas:

Já vi casos de uma menina de 15 anos ter vários miomas de tanto tomar a pílula do dia seguinte. (Nair, 30 anos, superior incompleto, casada).

Tem gente aí que não vai poder ter filho mais. A pílula do dia seguinte destruiu o útero dela. Vai enfraquecendo os hormônios todinhos. Não só os hormônios. Mas daqui a pouco vai consumindo os ossos. Se os hormônios ficarem ruins, fracos, os ossos também ficam. (Roberto, 42 anos, superior incompleto, casado).

O termo "hormônios" foi utilizado pela primeira vez em 1905, pelo professor de fisiologia Ernest H. Starling, referindo-se às mensagens químicas produzidas em determinados órgãos que, conduzidas pela corrente sanguínea, afetam outros sistemas e órgãos. De fato, a emergência desse conhecimento instaurou uma nova apreensão do corpo humano e sua regulação fisiológica. Uma concepção do corpo que se funda em parâmetros e medições hormonais, 
que se liga à medicina, mas também a outros campos disciplinares, sobretudo à bioquímica, tornou-se vigente (Harding, 1996; Oudshoorn, 1994).

Para Harding (1996, p. 99), a descoberta dos hormônios está entre as maiores invenções do século XX. Desde então, eles têm capturado as imaginações científicas e populares, por conta de seu poder de controlar a forma como cada indivíduo olha, sente e se comporta. Um novo modo de apreender os corpos femininos e masculinos emerge no conjunto desses novos saberes, a partir do que era conhecido em termos das gônadas humanas: os testículos e os ovários, interpretados como agentes de diferenças sexuais (Harding, 1996; Laqueur, 1992; Oudshoorn, 1994).

O receio que os entrevistados expressam sobre a contracepção de emergência está ligado, principalmente, à representação de que o medicamento contém altas dosagens hormonais. Essa concepção integra representações sociais mais amplas disseminadas sobre o método no contexto internacional, sendo possível observá-la em vários estudos com usuários e provedores dos serviços de saúde (Baldwin et al., 2008; Hussainy et al., 2011; Rocca et al., 2007; Shoveller et al., 2007). A mesma representação foi encontrada no Brasil, em pesquisa realizada com profissionais de saúde, em 2004, em quatro capitais brasileiras: Belém (Pará), Salvador (Bahia), Curitiba (Paraná) e Goiânia (Goiás) (Osis et al., 2006). Nesse estudo, pesquisadores observaram que a contracepção de emergência também foi concebida como um medicamento perigoso para a saúde das pessoas e, por isso, sua dispensação às usuárias sofria maior controle, permanecendo guardada em armários trancados com chaves e não acessíveis a qualquer funcionário.

Os temores de uma dose excessiva de hormônios são potencializados por uma concepção de que o método esteja sendo usado indiscriminadamente, de forma desmedida, repetitiva, em caráter regular e não emergencial, tal como refletido nas respostas dos participantes desse estudo. Uma balconista chega a expressar:

[...] vai acabar se tornando uma neosaldina [dipirona sódica]. (Rita, 24 anos, superior completo, solteira).

Teme-se uma apropriação indevida do método pelas mulheres, que possa causar prejuízos à saúde feminina ou o não cumprimento de uma conduta moral desejável, qual seja, primeiro planeja-se a reprodução, para depois se 
entregar aos deleites eróticos. O que se teme é o sexo sem controle por parte das mulheres. Não é aleatório que os motivos apontados pelos entrevistados para uso da contracepção de emergência evoquem atitudes de imprevidência, esquecimento, de não cuidado antecipado com a prevenção à gravidez, a exemplo de: as mulheres não utilizam outra forma de proteção $(78,4 \%)$, esquecem de usar o método hormonal de rotina $(59,7 \%)$, usam por displicência $(56,1 \%)$ ou imediatismo no ato sexual $(45 \%)$, por preguiça de usarem outro método $(34,7 \%)$ e insegurança quanto ao método adotado $(33,4 \%)$. Esse suposto comportamento feminino displicente, negligente com a contracepção antecipada, tem sido duramente criticado, apresentado como "sem-vergonha". Essa é a face moralizante da crítica ao método e por extensão às suas usuárias. Depoimentos obtidos na etnografia de Paiva (2014) e no trabalho de campo dessa pesquisa revelam o quão preconceituosos podem ser tais juízos de valor:

Acabou de fazer [sexo] e veio. [...] Aí tem gente que vem de cabelo molhado, pô. Acabou de sair de onde estava e vem aqui. (Teo, 30 anos, ensino superior incompleto, casado).

Elas dormem sem calcinha e acordam na drogaria. (Gilberto, 23 anos, ensino médio incompleto, solteiro - extraído de Paiva, 2014).

Tal como Fallon $(2010,2012)$ discute em seu trabalho com mulheres jovens inglesas, a sociedade explora a capacidade humana de sentir vergonha usando-a como uma espécie de agente civilizador, onde tal sentimento pode servir para produzir ou policiar certa moralidade que necessita em si de decodificação. $\mathrm{O}$ constrangimento percebido no balcão da farmácia, no momento da compra/venda da contracepção de emergência assinala que essa estratégia de produção social da vergonha surte efeitos precisos: $50,9 \%$ dos entrevistados notam que as consumidoras solicitam o medicamento nessas circunstâncias, ou seja, "envergonhadas".

Essa primeira chave de interpretação dos incômodos e resistências que a contracepção de emergência desperta nos sugere novos modos de atualização de uma biopolítica que por excelência busca o "governo da vida" (Clarke, 1998; Rabinow; Rose, 2006). Se a sexualidade estiver relativamente livre dos constrangimentos da reprodução, como controlaremos a vida de sujeitos, livres e autônomos, para decidirem o que fazer? 


\section{0 agenciamento feminino}

A segunda via de compreensão desse mal-estar que vimos discutindo em relação à contracepção de emergência sugere debruçarmo-nos sobre o agenciamento feminino que o uso desse método está explicitando. Trata-se da capacidade de autodeterminação dos sujeitos dentro de um campo de possibilidades (Cabral, 2011; Luker, 1975). A transformação dos modos de aproximação e interação sexual entre pares heterossexuais nas sociedades ocidentais contemporâneas torna o planejamento dos intercursos mais circunstancial. Dentre os motivos apontados pelos farmacêuticos para o uso do método surgiu a não manutenção de relações sexuais frequentes $(25,3 \%)$. Adotar o uso regular de pílula anticoncepcional sem a existência de um parceiro fixo não tem sido comumente frequente no contexto brasileiro (Cabral, 2011). O alto custo do preservativo feminino bem como o difícil acesso a ele nos serviços públicos de saúde não faculta o crescimento de seu uso. A contracepção de emergência, assim como o preservativo masculino, surgem como possibilidades extremamente viáveis em contextos de intercursos sexuais pouco frequentes e/ou não previstos. Tendo em vista os expressivos constrangimentos de gênero para uso do preservativo masculino em todas as relações sexuais, o método da contracepção de emergência surge como uma possibilidade muito mais viável às mulheres. Por um lado, ela oferta a "vantagem" de prescindir de uma negociação entre parceiros quanto ao uso do preservativo masculino. ${ }^{5}$ Por outro, ela também permite às usuárias superar as muitas dificuldades encontradas para acesso aos cuidados em saúde (consultas médicas, exames, atividades educativas de planejamento reprodutivo como pré-requisito para obtenção do método, disponibilidade de insumos) nos serviços públicos de saúde, sendo o recurso direto às farmácias a alternativa mais plausível para se obter a contracepção de emergência. Assim, tal estratégia feminina dispensa uma submissão aos poderes médico e de outras categorias profissionais (enfermeiros, assistentes sociais, farmacêuticos, agentes comunitários de saúde) que costumam inquirir e perscrutar sobre a vida sexual, afetiva e moral das usuárias, com vistas ao "aconselhamento" e "orientação" atinentes às praticas de saúde.

5 Não iremos nos deter aqui sobre as questões relativas à prevenção de DST/Aids e uso do preservativo masculino, por estar fora do escopo de nossa discussão.

Horizontes Antropológicos, Porto Alegre, ano 23, n. 47, p. 131-161, jan./abr. 2017 
Esse argumento nos permite discutir as lógicas sociais e simbólicas que possam estar presidindo o recurso à contracepção de emergência pelas mulheres, em especial as mais jovens, na acepção do que Fainzang (2003, p. 33) designa como "estratégias paradoxais". Como a autora assinala, "trata-se de estratégias adotadas, de maneira explícita, para resolver um problema de saúde e, de maneira implícita, para responder a outras necessidades, dando lugar a uma outra lógica". Decerto, a crescente difusão pública de informações de saúde, na esteira do que autores como Conrad (2007), Clarke et al. (2003), Williams, Martin e Gabe, (2011), Fainzang (2012, 2013) vem discutindo como desdobramentos de um intenso processo de medicalização nas sociedades contemporâneas, pode permitir usos sociais dos medicamentos (nesse caso, um contraceptivo hormonal) antes sequer cogitados. A via de reflexão que aposta no uso da contracepção de emergência como um dispositivo de afirmação da autonomia feminina pode revelar o quanto essa perspectiva pode estar ferindo hierarquias morais e sociais, de classe e de gênero entre nós. Não é casual que encontramos na pesquisa maciça rejeição entre balconistas e farmacêuticos à consulta sobre a disponibilidade da contracepção de emergência na modalidade OTC, ou seja, disponível nas gôndolas para livre acesso do cliente.

Seguindo uma perspectiva foucaultiana, a contracepção de emergência pode ser compreendida como um "dispositivo de sexualidade" (Foucault, 1999), que alude a estratégias de controle de si e de controle social, de disciplinamento dos corpos. Além disso, a contracepção de emergência é um artefato tecnológico, passível de prescrição por especialistas, de regulação pelas agências sanitárias, de comercialização controlada (OTC ou BTC) e de divulgação pelo marketing farmacêutico. No entanto, a utilização desse dispositivo de controle da potencialidade reprodutiva também remete ao agenciamento feminino sobre si na medida em que ele contém a possibilidade de a mulher prescindir das negociações e/ou da vigilância, seja com parceiros ou com as instituições de saúde.

Assim, duas questões se colocam de forma sinérgica: se, por um lado, o recurso à contracepção de emergência alude a certa autonomia feminina no controle de si e à possibilidade de escapar das negociações e controle frequentemente presentes no âmbito do par conjugal, por outro lado, ela é alvo de disputas institucionais diversas ao ser majoritariamente representada como algo arriscado e perigoso, uma "bomba hormonal". O debate sobre a contracepção 
de emergência coloca em relevo questões relativas ao domínio da moral e da sexualidade, sobretudo feminina, esfera historicamente submetida a diversos dispositivos de controle, ora mais ou menos explícitos.

As querelas em relação à contracepção de emergência sinalizam as múltiplas disputas em torno da sexualidade feminina. Captar e compreender os usos sociais que tal método contraceptivo possibilita nas interações sociais cotidianas nos permite elucidar novos modos de atualização de uma biopolítica contemporânea.

\section{Referências}

ARRAIS, P. S. D. et al. Perfil da automedicação no Brasil. Revista de Saúde Pública, São Paulo, v. 31, n. 1, p. 71-77, 1997.

BAJOS, N. et al. De la contraception à l'avortement: sociologie des grossesses non prévues. Paris: INSERM, 2002.

BAJOS, N. et al. Contraception: from accessibility to efficiency. Human Reproduction, Oxford, v. 18, n. 5, p. 994-999, 2003.

BALDWIN, S. et al. Who is using emergency contraception? Awareness and use of emergency contraception among California women and teens. Women's Health Issues, New York, v. 18, n. 5, p. 360-368, 2008.

BASTOS, L. L. O Consórcio Internacional sobre Contracepção de Emergência: um estudo dos argumentos para difusão dos contraceptivos de emergência em países em desenvolvimento. 2015. Dissertação (Mestrado em Saúde Coletiva)-Instituto de Estudos em Saúde Coletiva, Universidade Federal do Rio de Janeiro, Rio de Janeiro, 2015.

BERGALLO, P. El debate jurídico en torno a la anticoncepción de emergencia: una mirada comparada. In: ARILHA, M. et al. (Org.). Contracepção de emergência no Brasil e América Latina: dinâmicas políticas e direitos sexuais e reprodutivos. São Paulo: Oficina Editorial, 2010. p. 9-62.

BERGER, P. L.; LUCKMANN, T. A construção social da realidade: tratado de sociologia do conhecimento. 4. ed. Petrópolis: Vozes, 1978. 
BOZON, M. Sociologie de la sexualité. Paris: Nathan, 2002.

BOZON, M.; HEILBORN, M. L. Iniciação à sexualidade: modos de socialização, interações de gênero e trajetórias individuais. In: HEILBORN, M. L. et al. (Org.). O aprendizado da sexualidade: reprodução e trajetórias sociais de jovens brasileiros. Rio de Janeiro: Garamond: Fiocruz, 2006. p. 156-205.

BRANDÃO, E. R. Divulgação da contracepção de emergência na mídia impressa brasileira: necessidade do debate sobre sexualidade na adolescência. In: FIGUEIREDO, R.; BORGES, A. L. V.; BASTOS, S. (Org.). Panorama da contracepção de emergência no Brasil. São Paulo: Instituto de Saúde/SESSP: Escola de Enfermagem/USP: RedeCe, 2016. p. 211-233.

BRASIL. Decreto-lei $n^{\circ}$ 2848/40, de 7 de dezembro de 1940. Código Penal. Rio de Janeiro, 1940. Disponível em: <http://www.planalto.gov.br/ccivil_03/ decreto-lei/Del2848.htm>. Acesso em: 27 fev. 2016.

BRASIL. Lei $n^{\circ}$ 5.991, de 17 de dezembro de 1973. Dispõe sobre o controle sanitário do comércio de drogas, medicamentos, insumos farmacêuticos e correlatos, e dá outras providências. Brasília, 1973. Disponível em: $<$ http:// www.planalto.gov.br/ccivil_03/leis/L5991.htm>. Acesso em: 27 fev. 2016.

BRASIL. Ministério da Saúde. Secretaria de Política de Saúde. Área Técnica de Saúde da Mulher. Assistência em planejamento familiar: manual técnico. 4. ed. Brasília, 2002. (Série A: Normas e Manuais Técnicos n. 40).

BRASIL. Ministério da Saúde. Secretaria de Atenção à Saúde. Departamento de Ações Programáticas Estratégicas. Anticoncepção de emergência: perguntas e respostas para profissionais de saúde. Brasília, 2005.

BRASIL. Ministério da Saúde. Secretaria de Atenção a Saúde. Departamento de Ações Programáticas e Estratégicas. Nota técnica sobre anticoncepção de emergência. Brasília, 2006.

BRASIL. Ministério da Saúde. Pesquisa Nacional de Demografia e Saúde da Criança e da Mulher: PNDS 2006: relatório final. Brasília, 2008. 
BRASIL. Ministério da Saúde. Secretaria de Atenção à Saúde. Departamento de Ações Programáticas Estratégicas. Prevenção e tratamento dos agravos resultantes da violência sexual contra mulheres e adolescentes. Brasília, 2011a.

BRASIL. Ministério da Saúde. Política Nacional de Atenção Integral a Saúde da Mulher: princípios e diretrizes. Brasília, 2011b.

BRASIL. Ministério da Saúde. Protocolo para utilização do levonorgestrel na anticoncepção hormonal de emergência. Brasília, 2012.

CABRAL, C. S. Práticas contraceptivas e gestão da heterossexualidade: agência individual, contextos relacionais e gênero. 2011. Tese (Doutorado em Medicina Social)-Instituto de Medicina Social, Universidade do Estado do Rio de Janeiro, Rio de Janeiro, 2011.

CABRAL, C. S. Socialização contraceptiva e o aprendizado da sexualidade: uma abordagem socioantropológica do processo de construção da prática contraceptiva. 2012. Trabalho apresentado. XVIII Encontro Nacional de Estudos Populacionais, Águas de Lindoia, 19-23 de novembro de 2012.

CARRILLO-GARCÍA, C. et al. Influência do gênero e da idade: satisfação no trabalho de profissionais da saúde. Revista Latino-Americana de Enfermagem, Ribeirão Preto, v. 21, n. 6, p. 1314-20, 2013.

CLARKE, A. Disciplining reproduction: modernity, american life sciences, and "the problem of sex". Berkeley: University of California Press, 1998.

CLARKE, A. et al. Biomedicalization: technoscientific transformations of health, illness, and U.S. biomedicine. American Sociological Review, New York, v. 68, n. 2, p. 161-194, 2003.

CONRAD, P. The medicalization of society: on the transformation of human conditions into treatable disorders. Baltimore: JHU Press, 2007.

COSTA, N. F. P. et al. Acesso à anticoncepção de emergência: velhas barreiras e novas questões. Revista Brasileira de Ginecologia e Obstetrícia, Rio de Janeiro, v. 30, n. 2, p. 55-60, 2008. 
DIAS, T. M. Controvérsias e estabilização: o debate sobre as pílulas anticoncepcionais no diário O Globo, nas décadas de 1960 e 1970. 2015. Dissertação (Mestrado em Ciências)-Instituto de Saúde da Mulher, da Criança e do Adolescente Fernandes Figueira, Fundação Oswaldo Cruz, Rio de Janeiro, 2015.

DÍAZ, S. et al. Acceptability of emergency contraception in Brazil, Chile, and Mexico. 1 - Perceptions of emergency oral contraceptives. Cadernos de Saúde Pública, Rio de Janeiro, v. 19, n. 5, p. 1507-1517, $2003 \mathrm{a}$.

DÍAZ, S. et al. Acceptability of emergency contraception in Brazil, Chile, and Mexico. 2 - Facilitating factors versus obstacles. Cadernos de Saúde Pública, Rio de Janeiro, v. 19, n. 6, p. 1729-1737, 2003b.

DICIONÁRIO HOUAISS da língua portuguesa. Rio de Janeiro: Objetiva, 2012.

DOMINGUES, P. H. F. et al. Prevalência da automedicação na população adulta do Brasil: revisão sistemática. Revista de Saúde Pública, São Paulo, v. 49 , p. 1-8, 2015.

FAINZANG, S. Entre práticas simbólicas e recursos terapêuticos: as problemáticas de um itinerário de pesquisa. Antropolítica, Niterói, n. 15, p. 39-59, 2003.

FAINZANG, S. L'automédication ou les mirages de l'autonomie. Paris: Presses Universitaires de France, 2012.

FAINZANG, S. The other side of medicalization: self-medicalization and self-medication. Culture, Medicine and Psychiatry, Dordrecht, v. 37, n. 3, p. 488-504, 2013.

FALLON, D. Accessing emergency contraception: the role of friends in the adolescent experience. Sociology of Health \& Illness, Hoboken, v. 32, n. 5, p. $677-694,2010$.

FALLON, D. "They're gonna think it now": narratives of shame in the sexual health experiences of young people. Sociology, London, v. 47, n. 2, p. 318$332,2012$. 
FAÚNDES, A. et al. Emergency contraception under attack in Latin America: response of the medical establishment and civil society. Reproductive Health Matters, London, v. 15, n. 29, p. 130-138, 2007.

FAYA ROBLES, A. Da gravidez de "risco" às "maternidades de risco": biopolítica e regulações sanitárias nas experiências de mulheres de camadas populares de Recife. Physis: Revista de Saúde Coletiva, Rio de Janeiro, v. 25, n. 1, p. 139-169, 2015.

FOSTER, A. M.; WYNN, L. L. (Ed.). Emergency contraception: the story of a global reproductive health technology. New York: Palgrave Macmillan, 2012.

FOUCAULT, M. História da sexualidade I: a vontade de saber. 13. ed. Rio de Janeiro: Graal, 1999.

GAGNON, J. Uma interpretação dos desejos. Rio de Janeiro: Garamond, 2006.

GONÇALVES, H. et al. Contraceptive medicalisation, fear of infertility and teenage pregnancy in Brazil. Culture, Health \& Sexuality, London, v. 13, n. 2, p. 201-215, Feb. 2011.

HARDING, J. Sex and control: the hormonal body. Body \& Society, London, v. 2, n. 1, p. 99-111, 1996.

HARDY, E. et al. Anticoncepção de emergência no Brasil: facilitadores e barreiras. Cadernos de Saúde Pública, Rio de Janeiro, v. 17, n. 4, p. 10311035, 2001.

HUSSAINY, S. Y. et al. Provision of the emergency contraceptive pill without prescription: attitudes and practices of pharmacists in Australia. Contraception, Los Altos, v. 83, n. 2, p. 159-166, 2011.

JÜTTE, R. Contraception: a history. Cambridge: Polity Press, 2008.

KORNBLIT, A. L. (Coord.). Metodologías cualitativas em ciências sociales: modelos y procedimentos de análisis. 2. ed. Buenos Aires: Biblos, 2007.

LAQUEUR, T. Making sex: body and gender from the Greeks to Freud. Cambridge: Harvard University Press, 1992. 
LEAL, O. F. Sangue, fertilidade e práticas contraceptivas. In: ALVES, P. C.; MINAYO, M. C. S. (Org.). Saúde e doença: um olhar antropológico. Rio de Janeiro: Editora Fiocruz, 1994. p. 127-139.

LUKER, K. Taking chances: abortion and the decision not to contracept. Berkeley: University of California Press, 1975.

MACHADO, M. H. et al. (Org.). Análise da força de trabalho do setor saúde no Brasil: focalizando a feminização. Brasília: Ministério da Saúde, 2006.

MARKS, L. Sexual chemistry: a history of the contraceptive pill. New Haven: Yale University Press, 2001.

MARTIN, A. La anticoncepción de emergencia en América Latina y el Caribe. Revista Panamericana de Salud Pública, Washington, v. 16, n. 6, p. 424-431, 2004.

MARTIN, E. A mulher no corpo: uma análise cultural da reprodução. Rio de Janeiro: Garamond, 2006.

MCLAREN, A. Histoire de la contraception: de l'antiquité à nos jours. Paris: Agnès Vienot, 1996.

MINAYO, M. C. S. Análise qualitativa: teoria, passos e fidedignidade. Ciência \& Saúde Coletiva, Rio de Janeiro, v. 17, n. 3, p. 621-626, 2012.

NAVES, J. O. S. et al. Automedicação: uma abordagem qualitativa de suas motivações. Ciência \& Saúde Coletiva, Rio de Janeiro, v. 15, supl. 1, p. 17511762, 2010.

NUCCI, M. Seria a pílula anticoncepcional uma droga de estilo de vida? Ensaio sobre o atual processo de medicalização da sexualidade. Sexualidad, Salud y Sociedad, Rio de Janeiro, n. 10, p. 124-139, 2012.

OSIS, M. J. D. et al. Atenção ao planejamento familiar no Brasil hoje: reflexões sobre os resultados de uma pesquisa. Cadernos de Saúde Pública, Rio de Janeiro, v. 22, n. 11, p. 2481-2490, 2006.

OUDSHOORN, N. Beyond the natural body: an archeology of sex hormones. London: Routledge, 1994. 
PAIVA, S. P. Silêncio, não dito e vergonha no balcão da drogaria: estudo etnográfico sobre a comercialização da contracepção de emergência no Rio de Janeiro/RJ. 2014. Tese (Doutorado em Saúde Coletiva)-Instituto de Estudos em Saúde Coletiva, Universidade Federal do Rio de Janeiro, Rio de Janeiro, 2014.

PAIVA, S. P.; BRANDÃO, E. R. Contracepção de emergência no contexto das farmácias: revisão crítica de literatura. Physis, Rio de Janeiro, v. 22, n. 1, p. 17-34, 2012.

PECHENY,M.; TAMBURRINO, M.C. ¿"La palabra lo dice"? Interpretaciones cruzadas y obstáculos al acceso a la anticoncepción de emergencia. Sexualidad, Salud y Sociedad, Rio de Janeiro, n. 1, p. 158-176, 2009.

PEDRO, J. M. A trajetória da pílula anticoncepcional no Brasil (1960-1980). In: MONTEIRO, Y. N. (Org.). História da saúde: olhares e veredas. São Paulo: Instituto de Saúde - SES/SP, 2010. p. 141-157.

PERPÉTUO, I. H. O.; WONG, L. L. R. Desigualdade socioeconômica na utilização de métodos anticoncepcionais no Brasil: uma análise comparativa com base nas PNDS 1996 e 2006. In: BRASIL. Ministério da Saúde; Centro Brasileiro de Análise e Planejamento. Pesquisa Nacional de Demografia e Saúde da Criança e da Mulher - PNDS 2006: dimensões do processo reprodutivo e da saúde da criança. Brasília: Ministério da Saúde, 2009. p. $87-104$.

PIRES, A. P. Amostragem e pesquisa qualitativa: ensaio teórico e metodológico. In: POUPART, J. et al. A pesquisa qualitativa: enfoques epistemológicos e metodológicos. Petrópolis: Vozes, 2012. p. 154-211.

PRESCOTT, H. M. The morning after: a history of emergency contraception in the United States. New Jersey: Rutgers University Press, 2011.

RABINOW, P.; ROSE, N. O conceito de biopoder hoje. Política \& Trabalho: Revista de Ciências Sociais, João Pessoa, n. 24, p. 27-57, abr. 2006.

ROCCA, C. et al. Beyond access: acceptability, use and nonuse of emergency contraception among young women. American Journal of Obstetrics \& Gynecology, New York, v. 196, n. 1, p. 29, 2007. 
ROHDEN, F. O império dos hormônios e a construção da diferença entre os sexos. História, Ciências, Saúde - Manguinhos, Rio de Janeiro, v. 15, supl., p. 133-152, jun. 2008.

SCHUTZ, A. Atenção seletiva: relevâncias e tipificação. In: SCHUTZ, A. Fenomenologia e relações sociais. Rio de Janeiro: Zahar, 1979. p. 110-120.

SHOVELLER, J. et al. Identifying barriers to emergency contraception use among young women from various sociocultural groups in British Columbia, Canada. Perspectives on Sexual and Reproductive Health, New York, v. 39, n. 1, p. 13-20, 2007.

SILVERMAN, D. Analysing talk and text. In: DENZIN, N. K.; LINCOLN, Y. S. (Ed.). Handbook of qualitative research. 2 nd. ed. Thousand Oaks: Sage, 2000. p. 821-834.

SIMONDS, W.; ELLERTSON, C. Emergency contraception and morality: reflections of health care workers and clients. Social Science \& Medicine, Oxford, v. 58, p. 1285-1297, 2004.

SOUZA, R. A.; BRANDÃO, E. R. À sombra do aborto: o debate social sobre a anticoncepção de emergência na mídia impressa brasileira (2005-2009). Interface, Botucatu, v. 16, n. 40, p. 161-175, 2012.

SPENCER, G. et al. Examining the concept of choice in sexual health interventions for young people. Youth \& Society, Thousand Oaks, v. 46, n. 6, p. 756-778, 2014.

TEIXEIRA, M. et al. Representations and uses of emergency contraception in West Africa. A social anthropological reading of a northern medicinal product. Social Science \& Medicine, Oxford, n. 75, p. 148-155, 2012.

TONE, A. Devices and desires: a history of contraceptives in America. New York: Hill and Wang, 2001.

WATIER, P. Une introduction à la sociologie comprehensive. Paris: Circé, 2002. 
WATKINS, E. S. On the pill: a social history of oral contraceptives, 19501970. Baltimore: The John Hopkins University Press, 1998.

WATKINS, E. S. How the pill became a lifestyle drug. The pharmaceutical industry and birth control in the United States since 1960. American Journal of Public Health, Washington, v. 102, n. 8, p. 1462-1472, 2012.

WEEKS, J. O corpo e a sexualidade. In: LOURO, G. L. (Org.). O corpo educado: pedagogias da sexualidade. 2. ed. Belo Horizonte: Autêntica, 2000. p. 35-82.

WORLD HEALTH ORGANIZATION. Fact sheet on the safety of levonorgestrel-alone emergency contraceptive pills (LNG ECPs). Geneva, 2010. Disponível em: <http://whqlibdoc.who.int/hq/2010/WHO_RHR_ HRP_10.06_eng.pdf $>$. Acesso em: 30 set. 2014.

WORLD HEALTH ORGANIZATION. Emergency contraception: fact sheet n. 244. Geneva, July 2012. Disponível em: $<$ http://www.who.int/mediacentre/ factsheets/fs244/en/>. Acesso em: 27 fev. 2016.

WILLIAMS, S. J.; MARTIN, P.; GABE, J. The pharmaceuticalisation of society? A framework for analysis. Sociology of Health \& Illness, Hoboken, v. 33, n. 5, p. 710-725, 2011.

Recebido em: 28/02/2016

Aprovado em: 30/09/2016 